\title{
KELTEN UND GALLIER.
}

Die 'Keltenfrage' erfreut sich von jeher des Rufes ganz besonderer Schwierigkeit. Es hat sich im Laufe der Zeit das gläubig hingenommene Dogma herausgebildet, dass auf keltischem Gebiet die Schwierigkeiten, die jeder Urgeschichtsforschung nun einmal eigen sind, potenziert und durch anderwärts unbekannte vermehrt auftreten. In gewissem Sinne ist das auch zutreffend. Allein die Momente, die die Erkenntnis erschweren, sind grösstenteils nicht organisch, nicht in der Natur des Forschungsobjektes begründet, sondern sind erst hineingetragen worden. Die Tradition der Meinungen, um einen Ausdruck Virchows zu gebrauchen, hat hier mehr als irgendwo anders dazu beigetragen, die Thatsachen zu verdunkeln. Das hängt wieder damit zusammen, dass alles, was mit wirklichen oder vermeintlichen Kelten zu thun hat, eine ganz eigentümliche Anziehungskraft auf Dilettanten und Unberufene aller Schattierungen ausgeübt hat und noch ausübt. Die Rückwirkung auf die ernst zu nehmenden Forscher ist nicht ausgeblieben. Die Frage war zeitweise derartig verrufen, dass die Keltomanie in Keltophobie umschlug; eine nüchterne Auffassung schien unmöglich. Das ist nun freilich alles viel besser geworden. Die Erforschung der keltischen Sprachen und die sachgemässe Behandlung der Angaben der antiken Schriftsteller über die Kelten haben die Gemüter wesentlich beruhigt und das Chaos der widerstreitenden Ansichten gelichtet. Man hat erkannt, dass die 'Keltenfrage' im Grunde keine andern Schwierigkeiten aufweist als die 'Germanenfrage' oder die 'Griechenfrage'. Aber noch schleppt man sich mit mancher überkommenen Lehre, die vor einer strengeren Kritik nicht 
bestehen kann, mühsam herum oder glaubt gar an ihr einen Schatz zu besitzen. Ein derartiger Besitz von zweifelhaftem Werte scheint mir die von Alexandre Bertrand (Revue archéologique I, $1 \mathrm{ff}$., Archéologie celtique et gauloise ${ }^{2} 415$ ) in die Welt gesetzte Lehre, dass zwischen 'Kelten' und 'Galliern' ein tiefgreifender Unterschied bestanden habe. Sie soll mit ihren Konsequenzen geprüft werden. ${ }^{1}$ )

Hekataeus von Milet eröffnet den Reigen der griechischen

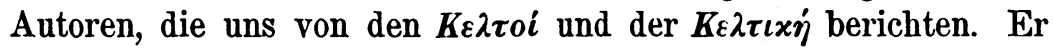

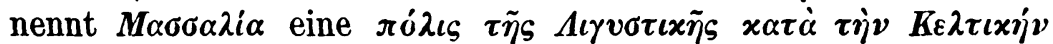
(fragm. hist. graec. I, s. 2, fragm. 22). Laut Stephanus von Byzanz macht er ferner eine $\pi \dot{\alpha}_{\lambda \iota \varsigma} K \varepsilon \lambda \tau \iota x \dot{\eta} N v_{\rho} \alpha \xi$ namhaft, die mit Ed. Meyer vermutlich in Spanien gesucht werden muss. Etwas

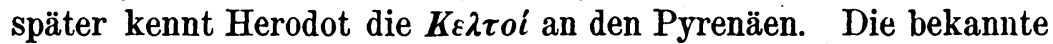

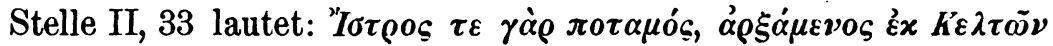

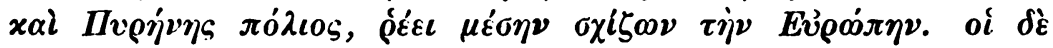

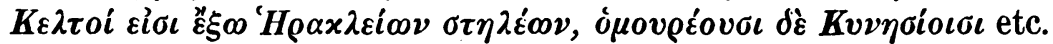
(die zweite Stelle IV, 49, vgl. auch Aristoteles meteorol. 1, 13, p. $350 \mathrm{~b}$ 2). Es war, nebenbei bemerkt, Al. Bertrand vorbehalten, aus dieser Stelle herauszulesen, dass die Kelten damals in der Gegend der wirklichen Donauquelle, also im südwestlichen Winkel Deutschlands, sassen (Les Celtes dans les vallées du Pô et du Danube 12). Im Anfang des vierten Jahrhunderts machten die Römer unliebsame Bekanntschaft mit keltischenn ${ }^{2}$ ) Scharen, die

1) Selbstverständlich haben sich viele Gelehrte von B.'s Hypothese niemals blenden lassen. Da diese aber entweder eine vornehme Zurückhaltung beobachteten oder, wie z. B. d'Arbois de Jubainville, auf die Argumente der Gegner nicht genügend eingingen, oder wie Lefèvre (Bulletins de la soc. d'anthropologie de Paris VI, 330 ff.) und Mortillet (Formation de la nation française bes. $92 \mathrm{ff}$.) Wahres und Falsches durcheinander mischten, musste die Irrlehre viele Opfer unter denen fordern, die an den Urgeschichtsproblemen gleichfalls arbeiten, aber den historischen und linguistischen Dingen ferner stehen. Die gemeinsame Arbeit der Historiker, Linguisten, Prähistoriker und Anthropologen kann sich aber nur dann gedeihlich gestalten, wenn ein wirklicher Austausch stattfindet. Für dieses grössere Publikum, an das sich B.'s Arbeiten wenden, ist auch diese ihre Kritik bestimmt. Ohne Wert ist eine deutsche Monographie über die Kelten, die 1891 in der Festschr. z. 50 jähr. Jub. d. Ver. d. Altertumsf. im Rheinl. S. 62 ff. erschienen ist und H. Schaafthausen zum Verfasser hat.

9) Im Hinblick auf die Vieldeutigkeit des Wortes 'Kelte', über die seinerzeit Broca herzbewegliche Klage gefuhrt hat (Revue d'anthropologie II), bemerke ich, dass für mich ein 'Kelte' schlechthin der Träger eines durch 
sich über Italien ergossen, um nach Eroberung Roms das Land nördlich des Apennin mit Beschlag zu belegen. Es waren formae hominum invisitatae (Livius V, 35). Die Römer nannten sie Galli. Dies ist für alle Zeiten die spezifisch römische Bezeichnung für alle festländischen Kelten mit Ausnahme der Belgen geblieben. Niemals werden die britannischen Kelten Galli genannt, obwohl ihre enge Verwandtschaft mit den Galli nicht verkannt wird. Etwa ein Jahrhundert nach der Schlacht an der Allia und dem Falle Roms tauchten die Kelten in der griechischen Kultursphäre auf. ') Die Griechen, die bis dahin nur den Namen

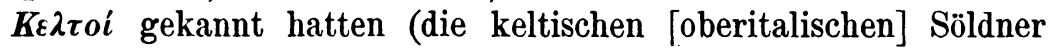
der Syrakusaner werden von Plato, die norditalischen Galli von

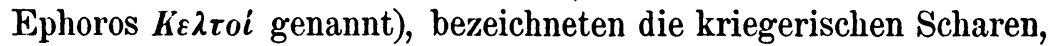
die bis Delphi vordrangen, als $\Gamma \alpha \lambda \alpha \dot{\tau} \alpha \iota$ (vgl. zu diesen Balkankelten und ihren Zügen Garofalo, Revue des études grecques $\mathrm{XIII}, 450 \mathrm{ff}$.). In der Folge blieb dieser Name an den im Balkan und weiter östlich sitzenden Kelten haften. Das ältere $K \varepsilon \lambda \tau o i$ diente weiter teils als Gesamtname, unter den auch die Germanen fielen, teils im engern Sinne als Bezeichnung der italischen, spanischen, französischen Kelten. Griechen, die über römische Geschichte schrieben, passten sich jedoch bis $\mathrm{zu}$ einem gewissen Grade dem römischen Sprachgebrauch an und nannten die italischen etc. Kelten $\Gamma \alpha \lambda \alpha \dot{\tau} \alpha \iota$, weil eben die Römer Galli sagten und beide Wörter (Galli und $\Gamma \alpha \lambda \alpha \dot{\alpha} \tau \alpha$ ) offenbar für identisch galten. Durch Caesar wurde schliesslich das alte griechische $K \varepsilon \lambda \tau$ oi gewissermassen neu entdeckt, allerdings in etwas abweichender Form, als Celtae. Die Celtae sind nach ihm die Bewohner des Landes zwischen Garumna und Sequana. Dies in grossen Zügen der Thatbestand. Welchen Anteil haben an dieser Buntheit einerseits der griechische und römische Sprachgebrauch, andererseits etwaige keltische Stammesverschiedenheiten?

Al. Bertrand glaubt nachweisen zu können, dass $K \varepsilon \lambda \tau o i$ und $\Gamma \alpha \lambda \alpha \dot{\tau} \alpha \iota$ bei Polybius zwei völlig verschiedene Völker sind. Unter

lautliche etc. Eigentümlichkeiten (Schwund von $p, e>\bar{\imath}$ ) scharf charakterisierten idg. Idioms ist.

1) Natürlich hatten schon vorher Berührungen stattgefunden. Diodor nennt XVII, 113, 12 unter den Völkern, die Gesandte an Alexander in Babylon

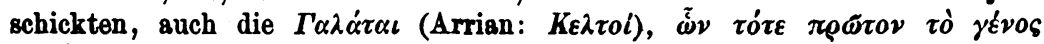

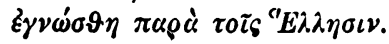




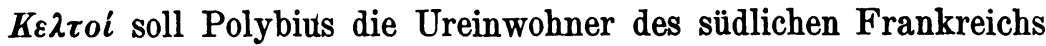
und des nördlichen Italiens verstehen, unter $\Gamma \alpha \lambda \alpha \dot{\tau} \tau \alpha \iota$ dagegen die kriegerischen Scharen, die Rom eroberten. Eine solche reinliche Scheidung besteht in Wirklichkeit nicht. Häufig genug

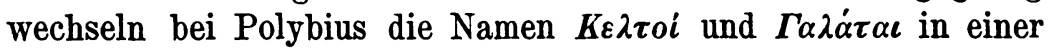
Weise, dass man sieht, sie sind für ihn gleichwertig. Die

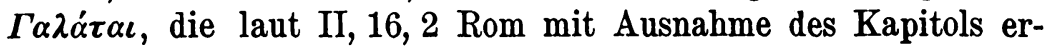

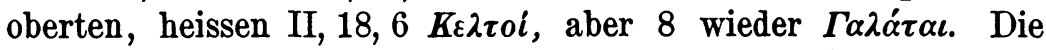
keltischen Völkerschaften Oberitaliens (also die $\alpha \dot{\pi} 0 \lambda \varepsilon \iota \varphi \vartheta \varepsilon \dot{\nu} \tau \varepsilon \varsigma$ $\tau \tilde{r}_{\varsigma} \sigma \tau \rho \alpha \tau \varepsilon i \alpha \varsigma$, wie sie bei Scylax peripl. 18 genannt werden) heissen bald $K \varepsilon \lambda \tau o i$, bald $\Gamma \alpha \lambda \alpha \dot{\tau} \alpha \iota$, die $\Gamma \alpha \iota \sigma \dot{\alpha} \tau \alpha \iota I^{\prime} \alpha \lambda \alpha^{\prime} \tau \alpha \iota$, die

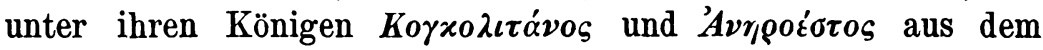
Rhonethal den italischen Stammesgenossen ${ }^{1}$ ) zu Hilfe kamen, II, 22, $8 K \varepsilon \lambda \tau \boldsymbol{L}^{\prime}$, die vereinigten Gaisaten, Boier und Insubrer heissen in den folgenden Kapiteln überwiegend $K \varepsilon \lambda \tau o i$, doch auch $\Gamma \alpha \lambda \dot{\alpha} \tau \alpha \iota(26,4)$. In K. von Beckers im übrigen ganz verfehlter Schrift 'Versuch einer Lösung der Celtenfrage' wird S. 16, 17 der nicht unebne Gedanke ausgesprochen, dass Pol. nur da $\Gamma \alpha \lambda \dot{\alpha} \tau \alpha \iota$ gebraucht, wo er aus einer römischen Quelle schöpft (ebenso Holder, Altcelt. Sprachschatz I, 892). Die Frage gehört vor das Forum der Quellenforschung (vgl. bisher über die Quellen der Polybianischen Darstellung der gallischen Kriege Niese, Hermes XIII, $410 \mathrm{ff}$., Nitzsch, Die römische Annalistik $271 \mathrm{ff}$.), vorläufig scheint es mir jedoch wahrscheinlicher, dass dem Historiker beide Namen gleich geläufig waren, und er je nach Laune bald diesen, bald jenen bevorzugte. ${ }^{2}$ ) Verhängnisvoll für die Folgezeit ist vor allem Bertrands Auffassung von Pol. II, 15, 1 ff. und 17, 9 ff. geworden. Aus beiden Stellen liest B. einen schroffen Gegensatz zwischen friedlichen, hochkultivierten Kelten und kriegerischen Galatern heraus (Bertrand et Reinach, Les Celtes dans les vallées du Pô et du Danube S. 29). 15, $1 \mathrm{ff}$. entwirft $P$. eine begeisterte Schilderung der gesegneten Poebene und des trefflichen Menschenschlages, der sie bewohnt. Er hat die Verhältnisse seiner Zeit im Auge. An der späteren Stelle hingegen beschreibt er die Vorfahren der oberitalischen Kelten

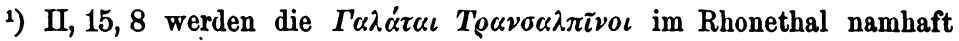

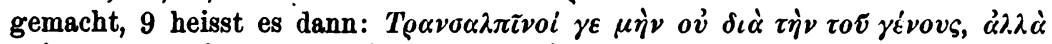

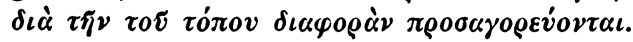

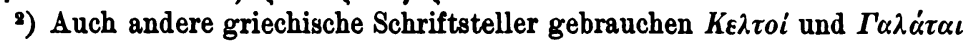
promiscue. 
des zweiten Jahrhunderts, die streitbaren Gesellen also, die den Römern zweihundert Jahre früher den gallischen Schrecken eingejagt hatten. Bei denen sah es freilich noch anders aus: g̈xovv

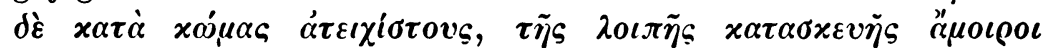

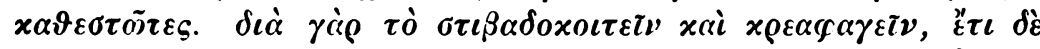

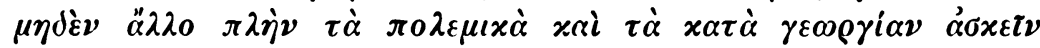

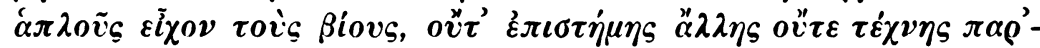

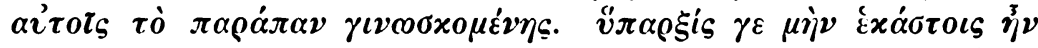

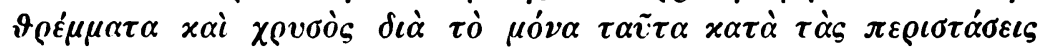

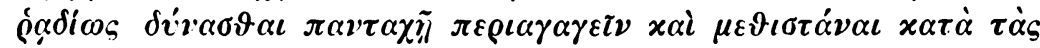

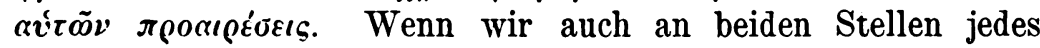
Wort für buchstäblich wahr halten, kann der Abstand zwischen einst und jetzt, wie ihn Pol. darlegt, keinen Unbefangenen befremden. Zwei Jahrhunderte sind an den bildungsfähigen Kelten natürlich nicht spurlos vorübergegangen. Tüchtige Krieger waren sie immer noch, aber sie überliessen es den Gaisaten, nach der Väter Sitte nackt in der vordersten Reihe zu kämpfen (II, 23, 8), was diesen übel bekam. Bertrand hält es für unmöglich, dass an beiden Stellen dasselbe Volk gemeint sei: es hat nach ihm in Italien friedliche Kelten gegeben, lange bevor die Galater einbrachen. Polybius weiss davon nichts, ebensowenig ein anderer Schriftsteller des Altertums. Eine ganz andere Frage ist es, wann und in welcher Form die keltische Invasion in Italien erfolgt ist. Bekanntlich giebt es über die Zeit des Einbruches eine zwiefache Überlieferung, die meisten Gelehrten haben sich für das spätere Datum (ca. 400) entschieden, doch findet auch das frühere (ca. 600) noch Verteidiger (zuletzt M. A. Pernice, Sui Celti e la loro immigrazione in Italia, vgl. dazu Revue Celtique $\mathrm{XX}, 576 \mathrm{ff}$.). Es scheint mir ausgemacht, dass die grosse Expansion der norditalischen Keltenstämme, die die Macht der Etrusker brach und Rom erschütterte, in die Wende des fünften Jahrhunderts fiel. $\mathrm{Zu}$ dieser Zeit mögen wirklich die meisten erst die Alpen überschritten haben. Es ist aber nicht einzusehen, warum nicht schon viel früher vereinzelte Scharen, kleinere Haufen, sich fast unbemerkt in der Poebene, deren Zustand damals natürlich ein wesentlich anderer war als einige Jahrhunderte später, eingefunden haben sollen. Man neigt dazu, sich grosse Völkerverschiebungen als einmalige Züge vorzustellen, während sie doch viel häufiger durch Summierung vieler kleiner Bewegungen zu Stande gekommen sind; äusserst lehrreich sind 
die Ausführungen Ratzels (Ber. săchs. Gesellsch. d. W., phil.-hist. Kl. 1898, 1 ff.), der diese Dinge mit dem weltweiten Blick des Anthropogeographen ansieht. Die Sage weiss zu berichten, dass ein Helvetier Helico, der in Rom das Schmiedehandwerk ausgeübt habe, mit Proben italischer Bodenerzeugnisse in die Heimat zurückgekehrt sei. Das habe den Anstoss zum Einbruch gegeben. Der Sagenform entkleidet hat die Erzählung ihren guten Sinn. Auch anderen antiken Schriftstellern entnehmen Bertrand und seine Gesinnungsgenossen ihre Beweise. Diodor sagt an der

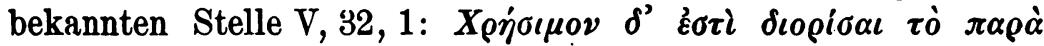

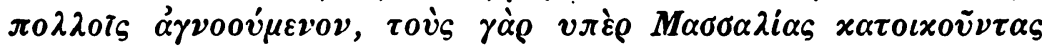

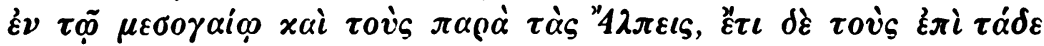

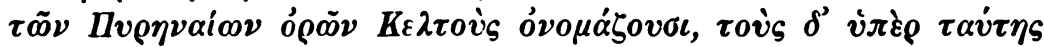

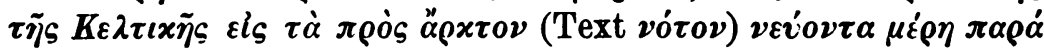

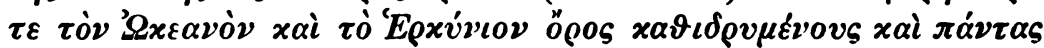

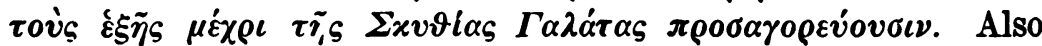
nördlich von den $K \varepsilon \lambda . \tau o i$ und den $K \varepsilon \lambda \tau \iota x \eta^{\prime}$ wohnen die $\Gamma \alpha \lambda \dot{\alpha} \tau \alpha \iota$ am Ocean und am hercynischen Gebirge bis zu den Skythen (Slaven) hin. Hier hat einmal Holtzmann recht gehabt, wenn er unter $\Gamma \alpha \lambda \dot{\alpha} \tau \alpha \iota$ die Germanen verstanden wissen wollte. Nur auf diese passt die Angabe über die Ausdehnung der $\Gamma \alpha \lambda \alpha \dot{\alpha} \tau \alpha \iota$. Germanen und Gallier warf man ja in älterer Zeit durcheinander. Die populäre römische Auffassung, der die bekannte Stelle des Strabo VII, 1, 2 Ausdruck verleiht, sah in den Germani 'germani Galli' (vgl. auch 0. Hirschfeld im Festgr. f. H. Kiepert). Zur Zeit Diodors war dieser Irrtum von den gut Unterrichteten überwunden, aber er klingt bei ihm eben in dem Gebrauche des Wortes $\Gamma \alpha \lambda \dot{\alpha} \tau \alpha \iota$ nach. An der Stelle V, 32 tritt somit die Erkenntnis von der Verschiedenheit der Germanen und Kelten in ganz eigenartiger, archaistischer Verkleidung auf, etwas anderes darf in ihr nicht gesucht werden. XXV, 13 berichtet derselbe

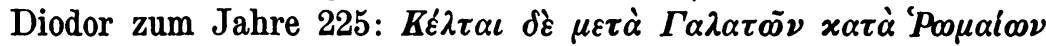

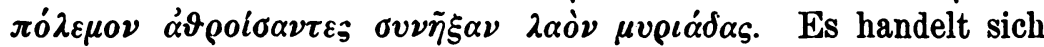
um den Krieg, den die norditalischen Kelten im Bunde mit transalpinen Stammesgenossen, den Gaisaten, gegen die Römer führten. Es sind mehrere Auffassungen möglich. Der Grieche konnte die transalpinen Kelten mit denen des Balkans verknüpfen und demgemäss $\Gamma \alpha \lambda \dot{\alpha} \tau \alpha \iota$ nennen, während er den ihm ferner stehenden italischen Kelten den älteren, gewissermassen unpersönlicheren

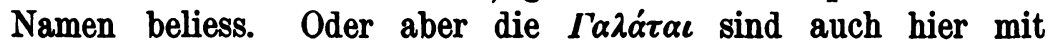


Germanen zu übersetzen. Bekanntlich erscheinen die unzweifelhaft in der Hauptsache keltischen Gaisaten in den kapitolinischen Fasten vom Jahre 222 als Germani (0. Hirschfeld, Hermes IX, 98, XI, 161, Mommsen RG. ${ }^{8}$ I, 555 Anm., Kossinna PBB. XX, 273 ff.). Dort sind sie deutlich durch spätere historische Spekulation hineingekommen. Vielleicht ist auch Diodor von dieser Ansicht beeinflusst. Darüber müssen die Historiker füglich das letzte Wort sprechen. $\mathrm{Zu}$ beachten ist übrigens noch, dass Diodor an der zuletzt angezogenen Stelle die Form $K^{\prime} \dot{\varepsilon} \tau \alpha \iota=$ lat. Celtae verwendet.

Sehr häufig ist ein Dialog des Sulpicius Severus in der Keltenfrage verwertet worden, von Bertrand Archéologie ${ }^{2}$ 416, Penka Origines Ariacae 106, Rhŷs Transactions Phil. Soc. 1891 -1893, S. 116, Rhind Lectures on Archaeology S. 7, um nur einige der neueren anzuführen. Bei Sulpicius Severus I, 26 sagt ein Gallier zu einem fein gebildeten Aquitanier: Sed cum cogito me hominem Gallum inter Aquitanos verba facturum, vereor ne offendat vestras nimium urbanas aures sermo rusticior. Der Aquitanier Postumianus antwortet darauf: Tu vero vel Celtice aut si mavis Gallice loquere, dum modo Martinum loquaris. Von einem Gegensatz zwischen Keltisch und Gallisch ist nicht die Rede. Postumianus sagt, um seine Worte etwas zu paraphrasieren, folgendes: Vor deinem schlechten Latein fürchte ich mich ganz und gar nicht. Ich will dir im Gegenteil noch eine weitere Konzession machen. Sprich meinetwegen keltisch oder gallisch, falls du diese Bezeichnung vorziehst; die Hauptsache ist, dass dn von Martinus sprichst. Keltisch und gallisch sind gleichwertig, etwa wie czechisch und böhmisch, wälsch und kymrisch u. dergl. Diese Deutung der Stelle, die allen Hypothesen den Boden entzieht, findet sich schon bei Windisch in Gröbers Grdr. d. rom. Phil. I, $297 \mathrm{f}$.

Rhŷs hielt sich besonders an den vermeintlichen Gegensatz zwischen keltischer und gallischer Sprache und beutete inn auf seine Weise aus. Die keltischen Sprachen teilen sich bekanntlich in der Behandlung der idg. labiovelaren Tenuis in zwei Gruppen. Die eine, ihr Hauptrepräsentant ist das Irische, hat idg. ku zunächst beibehalten (cruth aus *kurtu- Verf. KZ. XXXV, $253 \mathrm{ff}$.), dann zu reinem $c$ werden lassen. Die andere hat $k$ u $\mathbf{z u}$ gemacht. So das Britannische (kymr. pryd pedwar pwy) und in sehr zahlreichen Fällen das Keltische des Kontinents (Epona, 
Petrucorii etc.). Doch muss es auf dem Kontinent einst auch Vertreter der ersten Gruppe gegeben haben. Von jeher war der Name der Sequana und der Sequani ein Stein des Anstosses für die, denen das festländische Keltisch schlechthin als ältester Repräsentant des britannischen Zweiges galt. Rhŷs hat nun die Beispiele zu mehren gesucht. Vieles, was er vorbringt, ist unrichtig oder doch zum mindesten unsicher. $\left.{ }^{1}\right)$ Arquius ist auf der pyrenäischen Halbinsel mehrfach belegt. Aber Apilus Arquii CIL. II, 2433 macht uns schon an der Kelticität des Namens irre, da die Namen von Vater und Solun (Apilus dürfte keltisch sein) verschiedenen Dialekten angehören würden. Dass spanische Namen wie Aluquius (neben Allucius), Doquirus, Docquiricus keltisch sein müssen, kann ich nicht einsehen. Die Iberer waren doch auch noch da. Equabona kann eine hybride Bildung sein, falls es überhaupt richtig überliefert ist. Ins Gewicht fällt Quarquerni, Querquerni, der Name eines Stammes der keltiberischen Callaici (zum Wechsel $e: a$ vgl. Kossinna, IF. II, $181 \mathrm{f}$.), besonders wenn man das karnische Quarqueni Plin. III, 1302) und andrerseits Perperna, den Namen des berüchtigten Unterfeldherrn des Sertorius, daneben hält. Dagegen ist es wieder nichts mit Quassauna, das Rhŷs aus Oberitalien ins Feld führt. In Tiniatius Quasaunai CIL. V, 3463 ist gewiss der erste Name venetisch (ven. Tineh, freilich auch etrusk. Tinia, Tinu auf der Inschrift von Voltino), wahrscheinlich auch der zweite. Auch Querra dürfte den Venetern angehören, der Anklang an irisch Querai (Ogam, vgl. J. R. S. Antiq. of Ireland 1899, S. 402) ist bedeutungslos. Durch sein Suffix verrät sich Equasia, vgl. ven. (illyr.) Calsasia CIL. V, 2414, Audasius V, 3503. Die Quariates CIL. XII, 80 sind Ligurer; dass diese ein $q u$ besassen, bezeugt der echt ligurische Name Quiamelius mit der charakteristischen Bildung (vgl. Müllenhof, DA. ${ }^{2}$ III, $183 \mathrm{f}$.). Veiquasius sieht wieder venetisch aus. Es ist allerdings aus Piemont belegt, also westlich von der eigentlichen Venetersphäre (der Name der Venisami auf dem Bogen von Susa (CIL. V, 7231) klingt zwar stark venetisch, vgl. Venixama CIL. III, 3825, ist aber sicher ligurisch),

1) Vgl. auch d'Arbois de Jubainville, RC. XII, 477 f.; Loth im Krit. Jahresb. rom. Phil. IV, 1. Teil, S. $44 \mathrm{ff}$.

2) Nicht ganz sicher, vgl. jetzt Holder, Sprsch. II, 1057. Dort noch einige weitere ganz unsichere Fälle von $q u$. 
doch fällt das bei einem Einzelnamen nicht allzu schwer ins Gewicht. Dass schliesslich die ahd. Glosse Chorthonicum uualholant (Steinmeyer-Sievers III, 610) auf ein kontinentales Land Bezug habe, ist gänzlich unerweislich und unglaublich. Es ist klar, dass mit dem von Rhŷs zusammengetragenen Material nicht viel anzufangen ist. Auf festem Boden befindet mun sich dagegen bei den Sequanern, über deren Sprache der 1897 gefundene sogen. Kalender von Coligny (vgl. Thurneysen, Zs. f. celt. Phil. II, $523 \mathrm{ff}$.) doch wenigstens einiges Licht - es ist leider wenig genug - verbreitet hat. $\mathrm{Zu}$ dem Namen Sequani gesellt sich der Monatsname Equos ${ }^{1}$ ) und das freilich dunkle inquimon. Wenn neben diesen prinni, petiux mit offenbar aus qu entstandenem $p$ liegen, wird man das Auskunftsmittel Thurneysens S. 542 nicht verschmähen können. Die Sprache der Sequaner hätte somit weder zur $q u$ - noch zur $p$-Gruppe, sondern zu einer dritten vermittelnden gehört. Es geht aus all diesem hervor, auf wie schwachen Füssen die Annahme Rhŷs's steht, dass die Kelten des Festlandes gleichfalls in eine $q u$ - und $p$-Gruppe zerfallen seien. Wenn er aber vollends die $q u$-Gruppe mit den Celtae, die $p$-Gruppe mit den Galli identifiziert und aus der Severusstelle den Schluss zieht, dass damals beide Sprachen noch im lebendigen Gebrauche waren, so wird ihm darin kein Besonnener folgen. ${ }^{2}$ )

Wie zu erwarten war, haben sich auch die Prähistorie und die Anthropologie der Lehre vom Gegensatz der Kelten und Gallier bemächtigt. So hat ein ursprünglich auf die Polybiusinterpretation beschränkter Irrtum immer weitere Kreise gezogen und mit der Zeit das Bild von der vorgeschichtlichen Besiedelung nachmals keltischer Länder in unverantwortlicher Weise verfälscht. Da erfahrungsgemäss die Resultate der Prähistorie und Anthropologie von solchen, die in beiden Wissenschaften nicht selbständig mitarbeiten, meist mit vollkommener Skepsis oder aber mit gläubiger Ehrfurcht aufgenommen werden,

1) Epomanduodurum hiess eine Stadt im Lande der S., heute Mandeure, Dép. Doubs. Dies Epo- darf natürlich nicht gegen die oben ausgesprochene Anschauung geltend gemacht werden, da es einem Nachbardialekt, bezw. einem 'vorsequanischen' Dialekt angehört haben kann.

2) Rhŷs hält auch im Report of the Royal Commission on Land in Wales and Monmonthshire (London 1896) S. 66 an seiner Lehre fest, desleichen in dem Buche 'The Welsh People' S. 4. 
so ist nicht ausgeschlossen, dass die falsche Lehre auf diesem Umwege noch einmal wieder Geschichte wird. Da hătten wir den völligen Kreislauf der Dinge. Ein tüchtiger Ansatz dazu ist z. B. schon bei Rhŷs in dem eben citierten Report S. $66 \mathrm{zu}$ spüren. Dort wird die prähistorische Archäologie zum Zeugen dafür aufgerufen, dass die Kelten von den Galatern-Galliern sich durch die Bestattungsweise und Bewaffnung unterschieden. Die Kelten seien vielfach die Vorläufer der Galater-Gallier gewesen, welche im sechsten Jahrhundert der bis dahin keltischen Welt ein anderes Gepräge gegeben hätten. Die Kelten seien besonders im Alpengebiet, an der Donau und in Norditalien zu Haus, die Galater in Deutschland und Belgien. Wenn man hier überall die Namen weglässt, kommt schon eher etwas Vernünftiges heraus. Die Funde setzen uns in den Stand, gewisse Kulturkreise $z u$ bestimmen, übrigens meist auch nur dann, wenn ein Einzelobjekt zum Kriterium erhoben wird; der wertvollere Fall, wo die Abgrenzung auf Grund mehrerer Momente, vielleicht des Totalcharakters, stattfindet, ist bei weitem seltener. Aber selbst eine einheitliche materielle Kultur hat durchaus nicht ein einheitliches ethnisches Substrat zur Voraussetzung. Die Fundkarten ermöglichen es uns also nicht einmal die Grenzen anonymer Völker festzulegen. Die Namen vollends. kann natürlich nur die Geschichte liefern; wo diese versagt, bleibt die Prähistorie namenlos. So steht es in unserem Fall. Also fort mit allen Trugbildern!

Die Anthropologie ist eingestandnermassen kaum jemals in der Lage, ein historisches Problem, das sich an bestimmte Namen knüpft, zu lösen. Speziell in der Keltenfrage ist ihre Mitwirkung nicht von Segen gewesen. Die früher allgemein geteilte Ansicht, dass man in Frankreich und anderwärts zwischen kleinen, braunen, brachycephalen Kelten und grossen, blonden, dolichocephalen Galliern zu scheiden habe (z. B. Broca Bulletins de la société d'anthrop. de Paris, erste Serie, V, $457 \mathrm{ff}$.; Revue d'anthrop. II, 577; Lagneau, Artikel Celtes im Dictionnaire encycl. des sciences médicales; Penka, Origines ariacae 123), darf in dieser Formulierung als überwunden gelten (vgl. Collignons Ausführungen im Anschluss an den früher citierten Vortrag von Lefèvre), wenn auch z. B. Sergi (Atti della società Romana di antrop. III, 160 u. ö.), Humbert Molière (Introduction à l'histoire des Gaulois, Proto-Celtes, Celtes et Galates, S. 69) 
and Penka (Mitteil. d. anthrop. Ges. z. Wien XXVII, 18 ff.) daran testhalten.

Die Frage ist somit wieder auf dem Punkte, wo sie vor Bertrand war. Einen festen Anhalt scheint die bekannte, von Strabo, Livius, Plinius, Mela, Ammian wiederholte Angabe Cäsars zu bieten, dass die Bewohner des Landes zwischen Garumna und Sequana-Matrona in ihrer eigenen Sprache Celtae, auf lateinisch Galli hiessen (vgl. Wilkens, Quaestiones de Strabonis etc. fontibus, Marburger Diss. 1886, S. 31). Ob Cäsar diese Bemerkung aus seiner persönlichen Kenntnis des Landes schöpft, ob er sie einer litterarischen Quelle, etwa dem Posidonius, entnimmt, wissen wir nicht. Wir müssen sie einfach hinnehmen. Cäsar sagt Celta,

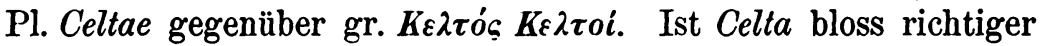
als $K \varepsilon \lambda \tau o ́ s$ oder von diesem verschieden? Miller, Strabos Quellen über Gallien und Britannien, S. 13 und Wilkens a. a. O. neigen sich letzterer Ansicht zu. Wenn man bedenkt, wie unbestimmt die erste Kunde gewesen sein mag, die vom Keltenvolke zu den Griechen drang, wird man die Möglichkeit, dass das richtige Geschlecht unterwegs abhanden gekommen ist, nicht rundweg ableugnen. Männliche $\bar{a}$-Stämme besass das Keltische ohne Zweifel (Holder I, 3, Stokes BB. XI, 154). Die Form Celta, die Cäsar einführt, flösst uns somit grösseres Zutrauen zu der Richtigkeit seiner Angabe ein. Müllenhoff (DA. I, 2167) geht in der Skepsis entschieden zu weit, wenn er daran zweifelt, dass die Kelten oder Teile derselben sich je selbst so genannt hätten. Er glaubt sogar, dass Celtillus, der Vater des Vercingetorix, seinen Namen nur den engen Beziehungen zu Massilia verdankt habe. Es fehlt nicht an inschriftlichen Zeugnissen für Namen, die Celt- enthalten. Celtillus ist aus Kaiser-Augst, Celtilla aus dem Rhonedelta überliefert, Cellinus aus dem Dép. Loire, Celto aus Haute Savoie, Ciltius aus Grenoble und Puy du Dôme. Die spanischen Celtiberi und Celtici sind sicher Kunstprodukte, aber Celti ist ein Ort auf dem rechten Ufer der Baetis, das Cognomen Celtitanus (zur Bildung vgl. Hübner, Ephem. epigr. II, S. 35) ist auf Inschriften und Münzen bezeugt, Celtus, Celta, Celtius sind mehrfach überliefert. Aus Italien liegen Celtus und Celtilia vor. Ein bekannter Ulsterheld heisst Celtchar macc Uthechair. Völkernamen werden zur Bildung von Personennamen verwendet, vgl. Boiorix, Boiocalus, ahd. Angilbreht,

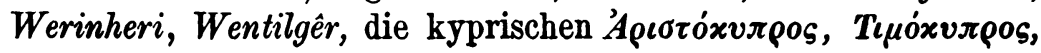




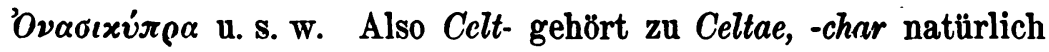

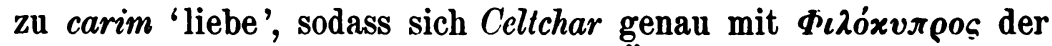
grossen Inschrift von Edalion deckt. Ü̉ber die genaue Bedeutung des Celt- wissen wir freilich nichts. Immerhin dürfte erwiesen sein, dass der Name Celtae mit echt keltischem Sprachgut zusammen hängt. $\mathrm{Ob}$ er jemals die Gesamtheit des keltischen Volkes bezeichnet hat, darf füglich bezweifelt werden. Solche Gesamtnamen pflegen sich verhältnismässig spät im Gefolge politischer Einigung oder doch festeren Zusammenschlusses einzustellen. Weshalb der Name Celtae gerade an den Bewohnern des Landes zwischen Garumna und Sequana haftet, wird niemals aufgeklärt werden, wenn uns nicht der Boden eines Tages eine Überraschung bereitet. In der Erwartung eines derartigen Fundes müssen wir uns darauf beschränken, den Beziehungen sprachlicher und anderer Art nachzuspüren, die zwischen den Celtae und den übrigen Kelten bestehen.

Es kann keinem Zweifel unterliegen, dass die italischen Kelten sich selbst den Namen Galli, die Donan- und Balkankelten den Namen $\Gamma a \lambda \alpha \dot{\alpha} \tau \alpha \iota$ beilegten. Mit ziemlicher Sicherheit dürfen ferner beide Namen identifiziert werden. $\Gamma \alpha \lambda \alpha \dot{\alpha} \tau \iota \iota$, gebildet wie

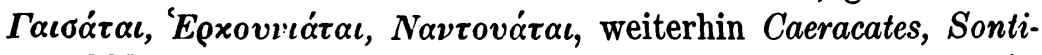
ates, Vellates u. dgl. gehört, wie man längst erkannt hat, zu mir. gal 'Tapferkeit', air. irgal 'Waffe', digal 'Rache' und bedeutet vermutlich 'Krieger, Held' (verwandt ist deutlich der Name der preussischen $\Gamma a \lambda i \nu \delta a \iota$ und der illyrischen $\Gamma \alpha \lambda \alpha \dot{\beta} \rho \iota 0 \iota)$. Ein Boierkönig des dritten Jahrhunderts hiess $\Gamma{ }^{\prime}{ }^{2} \alpha \tau \sigma_{S}$ (Pol. II, 21, 5); der Stammesname *Galatīni, in kymrischer Form Galedin, ist für England nur durch eine Triade bezeugt (Diefenbach, Celtica II, 72), für die Geschichte fällt in der Erzählung von den gwyr Galedin, welche yn y llongau moelion nach der Insel Wight kamen, nachdem ihr Land unter Wasser gesetzt war, nichts ab. Wie sich zu $\Gamma \alpha \lambda \alpha \dot{\alpha} \tau \iota$ lat. Galli verhält, ist nicht mit Sicherheit auszumachen. Man kann daran denken und hat daran gedacht, dass *Galati im Munde der Italiker zu Galli geworden sei, aber abgesehen von der Unwahrscheinlichkeit eines solchen Vorganges überhaupt, wird dieser spezielle Lautwandel durch die Lautgesetze keiner einzelnen italischen Sprache gerechtfertigt. Es müsste also der Name von einer Sprache zur andern weiter gegeben worden sein, was ja möglich ist, und dabei die Umformung erlitten haben. Der Name kann aber auch schon im 
Munde seiner keltischen Träger die historisch überlieferte Form angenommen haben. Galli wäre dann als Kurzform von $\Gamma \alpha \lambda a ́$ c $\alpha \iota$ aufzufassen.

Die Identität des Namens der italischen und der DonauBalkan-Kelten ist gewiss nicht zufällig. Die Einfälle in Italien und im Balkan stimmen nach Zeit und Charakter so genau zu einander, dass man hier wohl von einem ursprünglich einheitlichen Strome sprechen darf, der sich in zwei Arme geteilt hat. Hier erhebt sich nun gebieterisch die berühmte Frage, woher diese Menschenmassen gekommen sind. Allbekannt ist die Erzählung des Livius V, 34 und die Diskussion, die sich daran geknüpft hat und auch heute noch nicht zu völligem Abschluss gebracht ist. Livius leitet die italischen Kelten aus dem Lande der cäsarischen Celtae her. Sein Bericht enthält offenbar die heterogensten Bestandteile, die im einzelnen zu sondern auch der scharfsinnigsten Kritik nicht gelingen wird. Niese (Die keltischen Wanderungen, Zs. f. deutsches Altertum XLII, 129 ff.) bemisst den Wert der livianischen Erzählung sehr gering, er glaubt nicht einmal, dass in ihr eine echte (insubrische) Tradition verarbeitet worden ist, wie dies schon Müllenhoff angenommen hat. Nach Niese sind die Donauländer nördlich der Alpen die Heimat der Galli. Er geht, wie mir scheint, über die Namen der gallischen Völkerschaften etwas zu leicht hinweg. Faktisch sitzen doch im cäsarischen Gallien Lingones (Langres) und Senones (Sens) neben einander wie in Italien. Die Namensgleichheit allein will wenig besagen, sobald aber das geographische Moment hinzutritt, darf sie nicht mehr ohne weiteres beiseite geschoben werden. Der Fall kehrt ja beständig wieder.

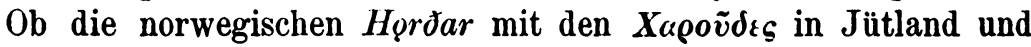
den Harudes des Ariovist mehr als den Namen gemeinsam haben, ist ungewiss; dass aber die Angeln, Sachsen und Jüten Englands mit denen des Kontinents genetisch zusammenhängen, unterliegt keinem Zweifel. Die Cenoman(n)i stellen in Frankreich eine Unterabteilung der Aulerci dar; bei ihnen, die nicht unmittelbare Nachbarn der Senonen sind, kann man schon eher an eine zufällige Namensgleichheit mit den italischen Cenomani denken.1) Was es mit der Angabe des Plinius (N. h. III, 130) Cenomanos

1) Die Verbindung wäre freilich hergestellt, wenn man die italischen Carni mit den französischen Car»uutes identifizieren dürfte. 
iuxta Massiliam habitasse in Volcis (auctor est Cato) für eine Bewandtnis hat, ist unklar. ${ }^{1}$ ) Nun liegen freilich zwischen dem Galliereinfall in Italien und der Erschliessung des Landes der Celtae mehrere Jahrhunderte, während welcher die verschiedensten Völkerverschiebungen stattgefunden haben können. Wir wissen also nicht, ob die Senonen und Lingonen schon lange da ansässig gewesen sind, wo sie Cäsar angetroffen hat. Wir wissen aber auch nichts, was eine solche Annahme unmöglich machte. Aus der Notiz Cäsars, BG. II, 4 (s. u.), folgt, auch wenn sie zuverlässig ist, keineswegs, dass Senonen und Lingonen einst im nachmaligen Belgien gesessen haben. Die Gallier, von deren Austreibung an der Cäsarstelle die Rede ist, können sich mit Verlust ihrer eigenen Stammesnamen unter die vom Belgiereinfall nicht betroffenen keltischen Völkerschaften an der Seine und Marne gemischt haben. So viel ist sicher, die Erzählung des Livius gewinnt ein wesentlich anderes Gesicht, wenn man sich die Lagerung der gleichnamigen Stämme hüben und drüben vergegenwärtigt. Es könnte doch sein, dass sie einen guten alten Kern enthielte. Seien wir doch ehrlich; wir wissen ja so wenig von den Völkerverhältnissen West- und Nordeuropas zur Zeit der Keltenzüge, dass es vermessen ist, sich mit Sicherheit für oder gar gegen den Bericht des Livius zu entscheiden. Hier kann einmal die Archäologie rettend einspringen. Mit den Galliern kam die La Tène-Kultur nach Oberitalien, charakterisiert vor allem durch den ganz eigenartigen Schwerttypus (vgl. Polybius II, 33, 3). Derselbe erfährt im Verlauf kleine Modifikationen. Das Schwert der Früh-La TèneZeit unterscheidet sich von dem später im ganzen Norden verbreiteten durch eine scharfe Spitze der Klinge. Solche Schwerter der ältesten La Tène-Zeit finden sich einerseits in der Champagne und im Nahe-Saargebiet, andererseits in Marzabotto (Tischler, Ber. üb. d. in d. physikal.-ökon. Ges. z. Königsberg geh. Vorträge, 1884 (XXV), S. 23).2) Wenn die Zukunft an diesem

1) Vgl. Müllenhoff, Deutsche Altertumsk. $\Pi^{2}, 260$.

2) Die Gräbcr der Champagne, die von Morel in seinem Werke 'La Champagne souterraine' beschrieben werden, stimmen überhanpt in ihrem ganzen Mobiliar anf das genaueste zu den gallischen Nekropolen, wie sie vor allem in und bei Bologna von Zannoni, Gozzadini und Brizio freigelegt worden sind; vgl. Brizio, Tombe e necropoli galliche della provincia di Bologna Bertrand et Reinach, Les Celtes $171 \mathrm{ff}$; Montelius, La civilisation primitive en Italie I, 356. 
Sachverhalt nichts ändert, fällt er schwer zu Gunsten der französischen Herkunft zum mindestens eines Teils der italischen Kelten in die Wagschale. Von dem Eigen- und Ortsnamenmaterial erwarte man keine Aufschlüsse. Alte Sonderbeziehungen treten in ihm nicht mehr zu Tage. Der sonstige Wort- und Formenschatz der italischen Kelten ist uns ja so gut wie unbekannt. Er giebt nur zu einer Bemerkung Anlass. Auf der Bilinguis von Todi erscheint zweimal eine Verbalform karnitu etwa in der Bedeutung congessit. Ihr gesellt sich das pluralische karnitus der Inschrift von Briona bei Novara im Gebiete der Insubrer. Eine entsprechende Bildung findet sich sonst nur auf einer patella aus Bavai, dem alten Bagacum der Nervier, die die Inschrift trägt: uritu Escingos (Mowat, Comptes rendus d. séances de l'Ac. des inscr. et belles-lettres, 4e série VIII, $250 \mathrm{ff}$.). Dies bedeutet offenbar 'Excingus fecit', uritı zu sonstigem ieuru, wobei auch die neukeltische Wortstellung zu beachten ist. Diese Übereinstimmung zwischen italischem und belgischem Keltisch ist an sich ein wichtiges Indicium, das allerdings durch den kläglichen Stand unserer Kenntnis des kontinentalen Keltisch überhaupt im Werte stark herabgesetzt wird. Was für Stämme sich sonst noch an dem italischen Zuge beteiligt haben, ist mit unseren jetzigen Mitteln nicht zu entscheiden. Eine der bekanntesten Begleiterscheinungen jeder grösseren Völkerbewegung ist das Mitgehen ursprünglich unbeteiligter Massen, die von dem Strome aus ihrem alten Zusammenhang losgerissen werden. Es widerspräche jeder Erfahrung, wollte man sich die Leute, die sich mit einem ad hoc angenommenen Gesamtnamen Galli $\Gamma \alpha \lambda \dot{\alpha} \tau \alpha \iota$ nannten, einheitlich vorstellen. Für die Ethnographie ist aus diesem Namen ebenso wenig zu gewinnen, wie aus dem der

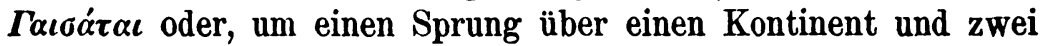
Jahrtausende zu wagen, der südafrikanischen Matabele.

Sicher scheint auch mir, dass der grosse Einbruch um 400 von Norden her erfolgt ist, nur suche ich im Gegensatz zu Niese den Ausgangspunkt anderwärts. Vermutlich hat nördlich der Alpen die Trennung der Galli und $\Gamma \alpha \lambda \alpha \dot{\tau} \tau \alpha \iota$ stattgefunden, jene gingen nach Süden, diese schwenkten nach Osten ab. Über die Herkunft dieser östlichen Galater sind wir zumeist völlig im Unklaren. Mit dem Namen der kleinasiatischen Tectosagen ist nichts anzufangen, dagegen können die $M_{\varepsilon}^{\prime} \lambda \delta \circ \iota$ in der Gegend von Sofia mit dem Vorort Meldia sehr wohl mit den Meldi im 
Dép. Seine-et-Marne zusammenhängen. Diese sind in einer Gegend ansässig, die im dringenden Verdachte steht, zu dem Zuge nach Osten Mannschaft geliefert zu haben. Sonstige Namensanklänge (vgl. die pannonischen Lingaustri : Lingones, Belgites : Belgae, Teurisci: Turones) sind für die geschichtliche Erkenntnis ohne jeden Wert.') Bekannt ist die Angabe des h. Hieronymus, Galatos propriam linguam eandem paene habere quam Treviros, die man sich nicht durch allzu kritische Bedenken verleiden lassen darf. Sie beweist jedoch nicht, dass vom Gallischen just die Sprache der Trevirer dem Galatischen am nächsten verwandt war, näher als etwa die alte Sprache der Pariser oder der Bewohner von Lyon. Hieronymus kannte entweder die Mundart von Trier besser als jede andere, oder er konnte nur sie vergleichen, da in Frankreich die alte Landessprache bereits ausgestorben war oder doch nur in Schichten

1) Dass Kelten schon im fünften vorchristlichen Jahrhundert in 0steuropa, nördlich der Karpathen, gesessen haben (Bremer, Pauls Grdr. d. germ. Philologie III, 781), ist unerweislich. Die Gleichung Nevooi (Herodot): Norici ist einer von den Einfällen, die die Sprachforschung bei den von anderen Disziplinen ausgehenden Urgeschichtsforschern nicht ganz ohne Grund in Misskredit gebracht haben. Bremer verlegt die Berührung von Kelten und Ostgermanen, deren sprachlicher Niederschlag die keltischen Lehnwörter im Gotischen (vor allem kelikn 'Turm') sind, zeitlich in das 5. oder 4. Jh. und örtlich in das Gebiet der oberen Weichsel. Er thut dies deshalb, weil nach seiner Ansicht die illyrisch-pannonischen Kelten längst romanisiert waren, als die Goten auf ihren Wanderungen ihr Gebiet passierten. So sicher ist das aber nicht. Nach dem oben angeführten Zeugnis des $h$. Hieronynus hatten die wahrlich stark exponierten Galater ihre heimische Sprache im 4. Jh. noch nicht eingebüsst. Aber davon ganz abgesehen wissen wir ja gar nicht, wie weit einst diese keltischen Lehnwörter in Ostgermanischen verbreitet waren. Die Goten können sie von verwandten Stämmen erhalten haben, die sie ihrerseits von Teilnehmern des Segovesuszuges - um mich der Terminologie des Livius zu bedienen - bezogen hatten. Im Gebiet der wandalischen Silingen

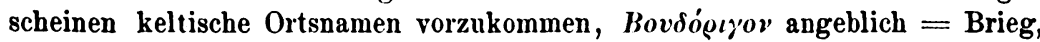

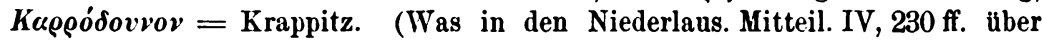
keltische Ortsnamen bei Görlitz vorgetragen wird, erinnert an die schönsten

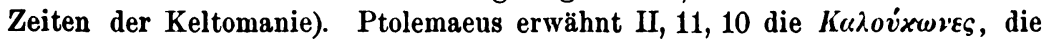
er íjò $\tau o \dot{v} \varsigma$ $\Sigma i \lambda \iota \gamma \gamma a \varsigma$ wohnen lässt. Da es auch in Raetia Calucones giebt, kann man hier an einen keltischen Stamm denken, der nur zum Teil den Übergang über die Alpen mitmachte. Alles dies zeigt, dass wir hinsichtlich einer Bezugsquelle für die keltischen Lehnwörter des Gotischen keineswegs in Verlegenheit sind, dass vielmehr weit eher ein embarras de richesse zu konstatieren ist. 
der Bevölkerung gesprochen wurde, mit denen der Mann der Kirche nicht in Berührung kam.

Die bisherigen Erörterungen hatten den Zweck, nachzuweisen, dass zwischen Celtae und Galli $\Gamma \alpha \lambda \alpha \dot{\alpha} \tau \iota \iota$ durchaus keine Kluft gähnt. Wie steht es nun mit den Belgae? Nach Cäsar zerfiel, wie bekannt, Gallien in drei Teile, die durch die Aquitani, Celtae und Belgae gebildet wurden. Hi omnes lingua, institutis, legibus inter se differunt. Richtiger sagt wohl Strabo IV, 1, 1:

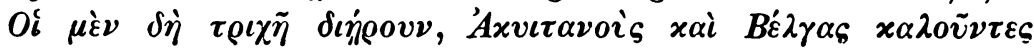

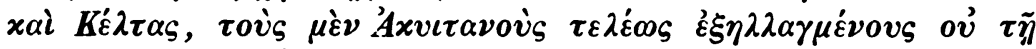

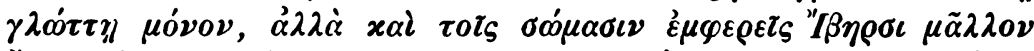

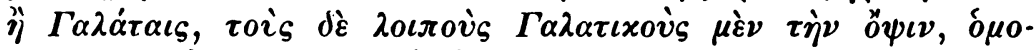

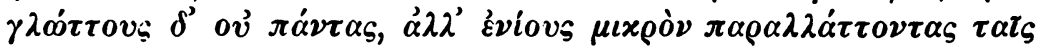

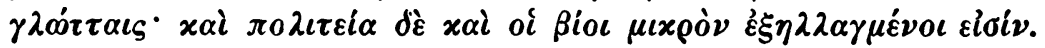
In sprachlicher Hinsicht werden Seine und Marne keine Grenze gebildet haben, hüben und drüben sprach man gleich, und erst die in beiden Richtungen entfernter Wohnenden unterschieden sich merklich. Deshalb konnte dennoch aus andern Gründen der Reisende, der die Flussgrenze passierte, den Eindruck erhalten, er befände sich in einem neuen Lande. Man denke etwa an Deutschland-Holland. Wir wissen ja nicht viel von der Sprache der Belgae, aber was wir wissen, berechtigt uns nicht, sie von der der Celtae zu trennen. Der Name Belgae hängt mit den pannonischen Belgites und dem spanischen Belgida zusammen, die Caturiges und Ceutrones kehren in Savoien wieder, die Suessiones erinnern an die spanischen Suessetani. Besonders deutlich sind die Beziehungen zu Britannien. Wie bekannt, haben Belgien und Südengland eine ganze Reihe von Stammesnamen gemein. Die Belgae selbst sind jenseits des Kanals bezeugt mit den Städten Venta, Iscalis, Aquae Sulis, ferner die Atrebates, die Catuvellauni. Der Name der Condrusi, die allerdings schon zu den linksrheinischen Germanen gehören, hat ein Seitenstück an dem britannischen Eigennamen Condraussius. Correus und Commius sind belgisch-britannisch; Andecombogius ist sonst nur aus der Inschrift von Briona bekannt, deren karnitus ja nach Belgien weist. Der Gott Camulus scheint besonders in Belgien und Britannien Verehrung genossen zu haben. Keltisch duro- bildet im allgemeinen in Zusammensetzungen den zweiten Teil, aber in Belgien und Britannien erscheint es an erster Stelle, vgl. Durocortorum Remorum und *Duroboium 
Durbuy auf dem Festland, Durobrivae, Durocornovium, Durolevum in England. Wenn Holder in deutlichem Bemühen, etwas für die Sprache der Belgae charakteristisches herauszufinden, den Ortsnamen Bratuspantium (im Gebiete der Bellovacer) 'mit noch nicht erklärtem $s p$ ' herausgreift (I, 374), beweist er keine glückliche Hand. Der Eigenname Atespatus zeigt ein analoges $s p$ auf echt 'celtischem' Boden (dép. de l'Allier). Wenn Bratuspantium unerklärt ist, so ist es doch nicht unerklärlich. Es ist vermittelst des Suffixes -io- von einem Eigennamen *Bratuspantos abgeleitet, der seinerseits wie Carantos participiale Bildung verrät. ${ }^{*}$ Bratuspantos führt auf das Participium eines denominativen Verbs zurück, dessen Ausgangspunkt etwa ein *bratuspo- war. Dies zerlegt sich sofort in *brātu- = air. bráth, k. brawd 'Urteil' und -spo-, die schwundstufige Kompositionsform der 'Wurzel' *sekn- 'sagen' (inseque, mk. heb), vgl. air. athesc 'Antwort', wozu das oben angeführte Atespatus, cosc, kymr. cosp 'Zurechtweisung'; *brātuspo- also 'Urteilsprecher' oder 'Urteilspruch', ein Wort von tadelloser Kelticität. Nun ist freilich die Ansicht vielfach verbreitet, dass das Keltentum der Belgen nur eine Tünche sei, unter der sich eine von Haus aus ganz verschiedene Volksart verberge. Diese Ansicht stützt sich auf die ja unzweifelhafte Thatsache, dass belgische Stämme, besonders die Nervier und Trevirer, sich ihres 'germanischen' Ursprungs rühmten, und dass die Völkerschaften der Eburones, Condrusi, Caeroesi u. s. w. sich direkt zum Gesamtnamen 'Germani (cisrhenani)' bekannten. Das rauhe, kriegerische Volkstum der Belgen, die der eindringenden römischen Kultur viel weniger Konzessionen gemacht haben als die Gallier, würde gut zu der Annahme eines verschiedenen Substrates stimmen. Nun ist garnicht daran zu zweifeln, dass diejenigen Keltenstämme, denen die Grenzwacht gegen die Germanen (in unserem Sinne) zugefallen war, einen gewissen Prozentsatz germanischer Elemente aufzuweisen hatten. Wo auch immer auf der Welt Völker bei einander wohnen, findet eine Abgabe des einen an das andere oder ein Austausch statt. Dass also die Belgen so manchen keltisierten Germanen zu den ihren gezählt haben, sei unseren Germanisten ohne weiteres zugegeben. Eine ganz andere Frage ist es aber, ob die alte Tradition vom germanischen Ursprung der Belgen wirklich dies besagen wollte, mit anderen Worten, ob die heutigen Germanisten recht daran thun, in Germani einen von 
den Kelten für die Deutschen - man gestatte mir in diesem Zusammenhang den Ausdruck - geprägten oder doch aufgegriffenen Namen zu erblicken. An der Aufhellung des Dunkels, das über dem Germanennamen liegt, ist auf germanistischer Seite mit unendlicher Mühe und grösstem Scharfsinn gearbeitet worden, ohne dass ein entsprechender Erfolg erzielt worden wäre. Wenn nicht ganz neues Material auftaucht, wird man über unsichere Aufstellungen nicht hinauskommen. Weder die berühmte Tacitusstelle, deren hoffnungslose Unklarheit oder Verderbtheit aller Anstrengungen spottet, noch irgend ein anderes der bekannten Inventarstücke der Germanenforschung vermag hier zu helfen. Thatsache bleibt, dass Stämme mit keltischen Namen westlich des Rheins den Gesamtnamen Germani führten, dass der Atrebate Commios Münzen mit der Legende (C)Garmano(s) Commios schlagen liess, dass die Abstammung von den Germanen ein Ruhmestitel war, dass Belgen und linksrheinische Germanen in älteren, einfacheren Zuständen lebten als die eigentlichen Gallier. Diese Data fügen sich verschiedenen Deutungen, vor allem immer noch der älteren, wonach Germani der Name der ostrheinischen Kelten oder doch einer bestimmten Gruppe derselben gewesen wäre. Es mag auch dem verfeinerten und sonst wohl zum Spotte über altväterische Sitten geneigten Gallier dunkel bewusst gewesen sein, dass die beste Kraft des Keltenvolkes in den streitbaren Stämmen lag, die unverdorben genug waren, sich ihrer Herkunft aus dem römischen Einfluss entrückten Lande rechts vom Rhein zu rühmen. Aber auch dies ist nur eine Hypothese. Nach Cäsar haben die Belgen in den neuen Sitzen westlich des Rheins Gallier vorgefunden und vertrieben. Man darf daraus schliessen, dass nicht immer Seine und Marne die Grenze der Celtae bildeten. Fassen wir Cäsars expulisse (BG. II, 4) wörtlich, so folgt daraus, dass die Belgen ihre unzweifelhaft keltische Sprache schon vom rechten Rheinufer mitgebracht haben. Die blosse Nähe der Gallier hätte unmöglich die Keltisierung der Belgen (und der linksrheinischen Germanen) bewirken können, deren Deutschtum somit immer nebelhafter wird. Gesetzt den (wahrscheinlichen) Fall, Cäsars expulisse sei nicht auf die Goldwage zu legen, sprechen dennoch alle Analogien dafür, dass die Belgen nicht erst westlich vom Rhein die keltische Sprache angenommen haben. Es darf bezweifelt werden, dass die Gallier trotz ihrer etwas höheren 
Kultur im Stande gewesen wären, das deutsche Volkstum ihrer Bezwinger mit Stumpf und Stiel auszurotten. Wo auch immer deutsche Eroberer inmitten eines ihnen an Kultur überlegenen Volkes ihre Nationalität und Sprache verloren haben, in Italien, Frankreich, Spanien, hat der Amalgamationsprozess, um von anderem zu schweigen, im Namenwörterbuch deutliche Spuren hinterlassen. Grade in vornehmen Geschlechtern sind deutsche Namen heimisch. Nichts entsprechendes findet sich bei Belgen und linksrheinischen Germanen. Auch die führenden Persönlichkeiten tragen unzweideutig keltische Namen.

Über die ethnische Stellung der britannischen Kelten sind wir nur sehr unvollkommen unterrichtet. Bekannt sind die Beziehungen zwischen Südengland und Belgien, die einst in der Vereinigung unter éinem Herrscher, Diviciacus, ihren Ausdruck gefunden haben. $\mathrm{Ob}$ die Parisii zwischen Humber und Tees von denen an der Seine abstammen, lässt sich nicht entscheiden, ebensowenig ob die irischen Mavájı mit den niederrheinischen Menapii zusammenhängen. Andere Namensanklänge verdienen keine Erwähnung. Die von Plinius n. h. IV, 104 angeführten festländischen Britanni bestätigt das Dorf Bretagne an der Mündung der Somme, doch ist sehr wohl denkbar, dass diese wie später die Bretonen erst durch eine rückläufige Bewegung auf das Festland herüber gedrängt worden sind. Ein eigentümlicher Missbrauch ist mit einer Stelle aus Prokops Gotenkrieg (IV, 20) getrieben worden. Prokop erzählt dort von einer

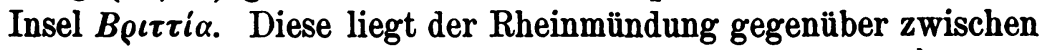
Britannien und Thule. Drei Stämme bewohnen sie, die 'A $\gamma \gamma$ i $20 \iota$,

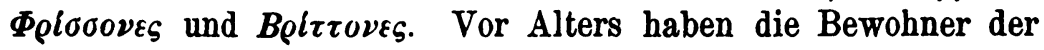
Insel eine Mauer gebaut, die sie in zwei Hälften teilt und zwar so gründlich, dass Luft, Boden und alles übrige in beiden verschieden ist. Es folgt darauf eine stimmungsvolle Sage von der nächtlichen Überfahrt der Toten. Natürlich ist diese Insel

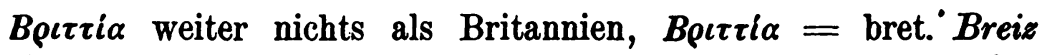
dio einheimische Namensform. Der von Rhŷs, Celtic Britain 2

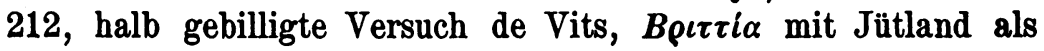
der Heimat der Brittones zu identifizieren, ist keiner ernsthaften Erwägung wert. Dem byzantinischen Historiker kann man

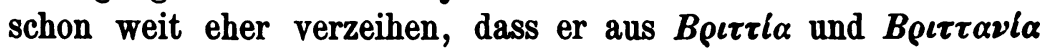
zwei verschiedene Länder machte.

In der Frage nach der Besiedelung Irlands und Englands 
durch die Kelten vertritt Rhŷs noch heute (Rhŷs and Brynmor Jones, The Welsh People 34/35) die Anschaungen, die uns aus seinen früheren Arbeiten (Report of the Royal Commission on Land in Wales and Monmouthshire Cap. VII, Rhind Lectures, Celtic Britain ${ }^{2}$ ) geläufig sind. Darnach hätten wir mit zwei Invasionen zu rechnen. Im sechsten oder fünften vorchristlichen Jahrhundert wären die dem $q u$-Zweige angehörigen Goidelen eingewandert, dreihundert Jahre später die Britten, die einen $p$-Dialekt sprachen. $\mathrm{Zu}$ diesem Ergebnis wird Rhŷs durch eine Wahrscheinlichkeitsrechnung geführt, die, wenn die in Betracht kommenden Faktoren etwas anders bewertet werden, ebenso gut ein völlig entgegengesetztes Resultat liefert. Der Umstand, dass die Goidelen den am weitesten nach Westen vorgeschobenen Posten der Inselkelten bilden, berechtigt noch keineswegs zu dem Schlusse, dass sie die ersten Ankömmlinge gewesen sind. Man kann ihre Sitze auch bei der Annahme verstehen, dass sie als die zuletzt gekommenen die dem Festlande näher liegenden Gegenden bereits okkupiert gefunden haben und daher am weitesten gewandert sind, ehe sich für sie ein Platz bot. Man denke an die Senonen in Italien. Zudem wissen wir nicht einmal mit Sicherheit, dass Irland seine keltische Bevölkerung ausschliesslich auf dem Wege über England erhalten hat. Alle Argumente, die man aus der Länge des Seeweges zwischen Frankreich und Irland $u$. dergl. zu schöpfen versucht sein kann, werden durch einen Hinweis auf die Besiedelungsverhältnisse in der Südsee erledigt. Die ganze Betrachtungsweise Rhŷs' leidet an dem bekannten Schematismus, der die Einheitlichkeit eines gegebenen Zustandes auch für seine Entstehung voraussetzt. Die Sonderung von $q u$ - und $p$-Kelten ist eine Thatsache der Sprachgeschichte, welche Rolle sie in der Besiedelungsgeschichte gespielt hat, ist unbekannt. Es ist nicht abzusehen, warum nicht $q u$ - und $p$-Kelten gelegentlich gemeinsam gewohnt haben und gewandert sein sollen. Die Not schweisst noch weit widerstrebendere Elemente zusammen. Gestehen wir also ehrlich ein, dass weder über die Art noch über die Zeit der Besiedelung Englands und Irlands irgend etwas Sicheres ausgesagt werden kann.

Ich breche diese kursorischen Ausführungen hier ab, denn der rote Faden, der sich durch sie hindurch zieht, ist nachgerade so dünn geworden, dass es Mühe macht, ihn überhaupt 
wahrzunehmen. In der keltischen Ethnographie ist recht vieles dunkel. Es begegnen uns da Gesamtnamen aller Art, deren Entstehung und Bedeutung wir nicht erraten können. Wenn es aber zwei Namen giebt, von denen wir mit gutem Gewissen sagen können, dass sie keinen alten Gegensatz enthalten, so sind es die, mit denen man ein so gewaltthätiges Spiel getrieben hat: Kelten und Gallier.

Berlin, Friedenau.

E. Zupitza. 